\title{
La gestion de la faune sauvage : une approche interdisciplinaire, une démarche adaptative
}

Quelles natures voulons-nous ? Quelle nature auronsnous? disait en 2001 l'appel à communications d'un colloque du programme Environnement-Vie-Sociétés du CNRS. De même aujourd'hui l'Office national de la chasse et de la faune sauvage se préoccupe de la, ou plutôt des gestions durables des espèces animales. Le premier paradoxe, c'est que la faune sauvage, qu'on se représente pourtant comme non soumise à l'homme, par opposition aux animaux domestiques directement sous le contrôle de l'homme, doit être gérée. Cette gestion donne lieu à controverse, car elle renvoie à notre question initiale : quelles natures voulons-nous? Et ce nous est pluriel. La difficulté que l'on affronte est donc de gérer dans un même temps la diversité biologique et la diversité culturelle. Gérer des écosystèmes et des interactions entre espèces et milieux n'est pas simple, puisque l'état de l'un dépend de la présence ou de l'état de l'autre et que cette gestion doit être envisagée selon les espèces à des échelles de temps ou d'espace radicalement différentes. Tenter de concilier les représentations contradictoires que se font de la nature les pêcheurs, les chasseurs, les touristes, les environnementalistes, les éleveurs, les agriculteurs tient aussi de la gageure dans une société démocratique et complexe. Tenir compte dans un même temps de tous ces facteurs demande en effet un effort interdisciplinaire que l'état d'avancement de la biologie de la conservation ne peut pas toujours fournir.

Que l'on protège une espèce ou que l'on souhaite en limiter les effectifs, voire l'éradiquer parce qu'elle est classée dans la catégorie "espèce invasive ", qu'on l'introduise ou la réintroduise, tous les milieux et espèces sont intégrés dans un plan de gestion et d'aménagement $\mathrm{du}$ territoire. Cette gestion est à la fois d'ordre biologique puisqu'il s'agit du domaine du vivant, d'ordre juridique, et en lien avec les nouveaux enjeux mis en avant par la Communauté européenne en matière d'espaces protégés, et d'ordre socioanthropologique, la nature étant utilisée, transformée, et pensée par des acteurs sociaux qui n'ont pas tous le même agenda.
Pourtant politiques, scientifiques et gestionnaires se trouvent souvent contraints, face à la société civile, à minimiser le facteur choix, c'est-à-dire la dimension politique de leurs actes de gestion. Car dès que ces acteurs admettent avoir effectué un choix, la controverse peut éclater : les citoyens qui auraient préféré une autre décision peuvent contester celle qui est prise. Le domaine de la faune sauvage est le lieu par excellence de la controverse publique. Les avis sont partagés et les camps violemment opposés : chasseurs contre protecteurs, ou « amis » contre détracteurs du loup ou de l'ours, pour citer les dossiers les plus en vue. Et la politique de concertation a ses limites, les oppositions se révélant parfois irréductibles. Pour échapper à la vindicte publique, les politiques, les environnementalistes ou les biologistes qui veulent protéger ou réintroduire une espèce sauvage que d'autres ne peuvent tolérer, tentent d'échapper par la naturalisation ( $c f$. le forum de S. Bobbé et A. Micoud dans ce numéro).

Pour comprendre ce qu'est la naturalisation, telle qu'elle a été mise en évidence par Bourdieu, rappelons la plus évidente et la mieux analysée de ces naturalisations, celle des relations homme-femme. La position dominante des hommes sur les femmes dans une société est souvent présentée en effet, non pas comme ce qu'elle est, un processus historique, un code culturel arbitraire et donc contingent, mais comme "naturelle». La naturalisation est toujours, quel que soit son objet, une tentative de soustraire la question naturalisée du domaine du politique en la déclarant inévitable et nécessaire. On assiste à un phénomène semblable dans le domaine de la gestion de la faune sauvage. Les politiques, scientifiques ou gestionnaires, plutôt que d'assumer leurs choix, s'affirment contraints à ne faire que ce qui est indispensable pour que la nature reste naturelle. Ils font alors mine d'oublier que nous vivons en Europe dans un monde transformé par la société, une nature anthropisée où l'apparition ou la disparition d'une espèce sont précisément liées à l'action de l'homme et à ses décisions. 
Prenons l'exemple de la campagne récente du ministère de l'Écologie et du Développement durable à propos de la restauration de la population des ours dans les Pyrénées. Pour ne pas prêter flanc à la controverse, Nelly Olin, en charge de ce ministère, minimise l'action en cours en n'utilisant pas de mots qui fâchent : «Il ne s'agit, répétons-le, que d'un renforcement d'une espèce déjà présente, pas d'une réintroduction ex nihilo ». Les précisions qu'elle apporte tendent à éluder toute responsabilité. Loin d'avoir pris une décision, elle s'est livrée à une simple action technique : «remplacer les trois ours morts par accident», puis « compenser très partiellement les naissances d'ours qui n'ont pas eu lieu du fait de ces disparitions prématurées ». Le dossier de presse du 13 mars 2006 justifie la restauration en affirmant qu'il « est montré scientifiquement que l'apport d'ours provenant d'une autre population viable est un impératif si l'on souhaite préserver la présence de l'espèce sur le territoire pyrénéen ». Si le choix est évoqué («si l'on souhaite»), l'argumentation («montré scientifiquement», et surtout, «impératif ») renvoie le poids de la décision sur les scientifiques.

Se pose également la question de l'état de référence invoqué dans les actions de restauration, souvent de façon très schématique, comme s'il n'y avait qu'un seul «avant ». L'état de référence doit être défini scientifiquement, mais également choisi socialement ou imposé juridiquement : veut-on restaurer une population ou un écosystème tels qu'ils étaient il y a un siècle, un millénaire, ou bien il y a quelques décennies? Si l'état désiré était le résultat d'un équilibre entre un certain milieu et certaines actions anthropiques, il faut admettre que $l^{\prime}$ on demande au génie écologique de se substituer à l'action d'une société passée. Il ne faut donc pas oublier que l'état de référence est un choix, même s'il est raisonné et instruit par les données scientifiques.

Le numéro que nous présentons ici, fondé principalement sur des communications présentées lors du colloque : "Gestions durables des espèces animales (mammifères, oiseaux) - approches biologiques, juridiques et sociologiques » organisé en 2004 par l'Office national de la chasse et de la faune sauvage ${ }^{1}$, tente un renversement critique : remplacer la naturalisation des questions gênantes par une analyse critique et interdisciplinaire. Il montre comment une espèce, en passant d'un statut à un autre, appelle des décisions tout à fait différentes, l'éradiquer ou l'admirer (C. Mougenot et L. Roussel). Deux auteurs (I. Mauz, F. Sarrazin) mettent en évidence le fait que si introductions et réintroductions ont beaucoup en commun, le sociologue ne voit pas les choses du même œil que le biologiste. Plusieurs textes analysent l'évolution du droit au fur et à mesure du changement sociétal des représentations de la nature. Quand les animaux sauvages qui, auparavant, pouvaient être chassés librement puisqu'ils n'appartenaient à personne, deviennent patrimoine commun, le droit se transforme, et continuera à le faire, pour rendre compte d'une nouvelle relation entre les êtres humains, les êtres non humains et les sociétés.

Prendre en compte la sauvegarde de la biodiversité dans les projets des sociétés humaines n'oblige-t-elle pas à adopter une démarche de gestion adaptative de la faune sauvage avec tous les protagonistes? Ne doit-on pas accepter dans une certaine mesure la démarche "essaierreur-ajustement » (expérimentation) dans les actions mises en place, compte tenu des incertitudes scientifiques dues à la fois à la complexité des sociétés humaines (diversités culturelles) et de la biodiversité (inertie intraspécifique due aux fonctionnements démographiques des populations et interactions interspécifiques liées au fonctionnement des écosystèmes) et aux aléas extérieurs aux systèmes non maîtrisés (une partie des changements globaux)?

Nous espérons que les contributions présentées dans ce numéro ${ }^{2}$ vous apporteront des éléments de réponse à ces questions, et vous en souhaitons bonne lecture.

Pierre Migot ${ }^{3}$ et Marie Roué ${ }^{4}$

\footnotetext{
1 Pour une présentation exhaustive de ce colloque, on se référera au compte rendu de P. Migot dans ce numéro.

2 Nous remercions les auteurs des articles pour leur travail effectué, en adaptant leurs communications présentées lors du colloque aux demandes des relecteurs et aux exigences de forme des rubriques de la revue, ainsi qu'à Robert Barbault pour sa contribution originale à ce numéro. Nous remercions enfin Marcel Jollivet et Henri Décamps pour la relecture des articles, le Comité de rédaction de NSS pour son soutien, Marie-José Leroy pour sa collaboration dans l'élaboration de la rubrique Lectures et Sylvie Zasser Bedoya pour le suivi de la réalisation de ce numéro spécial.

3 Biologiste, Directeur adjoint des études et de la recherche, Office national de la chasse et de la faune sauvage.

4 Anthropologue, Directrice de recherche CNRS, Muséum national d'histoire naturelle, Département "Hommes, natures,
} sociétés ». 